\title{
Computer-guidance in oral and maxillofacial surgery
}

\author{
Won Lee, D.D.S., M.S.D., Ph.D. ${ }^{1,2}$ \\ ${ }^{I}$ Department of Dentistry, School of Medicine, The Catholic University of Korea, Seoul, \\ ${ }^{2}$ Department of Oral and Maxillofacial Surgery, Dental Clinic, Uijeongbu St. Mary's Hospital, Uijeongbu, Korea
}

We cannot talk about computer-assisted practice without considering recent trends in dentistry. Every dental unit chair can now have its own computer and monitor after the introduction of digital imaging systems. Dental cone-beam computed tomography is now common in dental clinics ${ }^{1}$ due to its lower cost and smaller size than conventional computed tomography. Use of digital data, reformatted by computer, also helps in diagnosis and treatment plans. Oral and maxillofacial surgeons had been more familiar with digital imaging than most other dentists because they trained at hospitals. However, today, all dentists can use computerassisted equipment for diagnosis, planning, and treatment. Also, the 'hottest' products at current dental equipment exhibitions are computer-aided design/computer-aided manufacturing (CAD/CAM) hardware, software, and related equipment. Indeed, th ere is little in the latest equipment that does not have some degree of computer assistance.

$\mathrm{CAD} / \mathrm{CAM}$ systems have long been used in restorative and prosthetic dentistry ${ }^{2}$. Moreover, CAD/CAM equipment is a basic fixture in laboratories and some large dental clinics. A dentist can scan a patient's oral cavity with a digital scanner and prepare structures directly with a CAD/CAM system installed in their own clinic. This may allow a one-visit restoration with an inlay and/or crown for a patient.

Today, new three-dimensional diagnostic and treatment planning technologies have expanded its applications to implant dentistry ${ }^{3-5}$. A computer-generated virtual treatment plan is now a reality for accurate and predictable placement of implants. CAD/CAM abutments have the advantages of being specific to each patient and providing a better fit than ready-made abutments. To improve accuracy during implant placement, dentists also use stereolithography to manufacture CAD/CAM surgical templates. It allows the dentist to perform minimally invasive surgery without a flap, and to deliver the prosthesis to achieve immediate functional loading of the implant(s). Dental implant diagnosis and treatment have been revolutionized by recent advances in three-dimensional imaging, implant planning software, and associated surgical instrumentation. Use of computers can lead to better care and outcomes.

In the field of oral and maxillofacial surgery, computer applications can be used for imaging, planning the surgery, and guiding the operation ${ }^{6}$. Ultimately, this will lead to robotassisted surgery. Already, many robotic medical surgical procedures have been developed, especially for head and neck cancers ${ }^{7}$. In recent years, its use has been widened to orthognathic surgery ${ }^{8,9}$. Although robotic surgery in oral and maxillofacial surgery is not yet a common procedure, this results from costs and efficiency rather than technical difficulties. The introduction of robotic surgery to oral and maxillofacial surgeons who work with intraoral hard tissue could lead to dramatic results.

The effect of the computer in the field of dentistry has been increasing. In the near future, use of computer-assisted robotic surgical procedures in the field of oral and maxillofacial surgery will increase. Soon dentists will diagnose from digital images, make a surgical plan using a computer, and operate using robotics. Computer-assisted robot surgery will come, so oral and maxillofacial surgeons should be alert and keep up with developments in this technology. We surgeons working in Korea should take the lead in computer-assisted surgery, particularly robotic surgery technology, and collaborate with leaders in the global information technology industry.

\section{References}

1. Agrawal JM, Agrawal MS, Nanjannawar LG, Parushetti AD. CBCT in orthodontics: the wave of future. J Contemp Dent Pract 2013;14:153-7.

2. Fasbinder D. Using digital technology to enhance restorative dentistry. Compend Contin Educ Dent 2012;33:666-8.

3. Fuster-Torres MA, Albalat-Estela S, Alcañiz-Raya M, PeñarrochaDiago M. CAD / CAM dental systems in implant dentistry: update. Med Oral Patol Oral Cir Bucal 2009;14:E141-5.

4. Chiarelli T, Lamma E, Sansoni T. A fully 3D work context for oral implant planning and simulation. Int J Comput Assist Radiol Surg 2010;5:57-67. 
5. Orentlicher G, Abboud M. Guided surgery for implant therapy. Oral Maxillofac Surg Clin North Am 2011;23:239-56.

6. Nocini PF, Albanese M, Castellani R, Zanotti G, Canton L, Bissolotti G, et al. Application of the "All-on-Four" concept and guided surgery in a mandible treated with a free vascularized fibula flap. J Craniofac Surg 2012;23:e628-31.

7. Park YM, Kim WS, Byeon HK, De Virgilio A, Lee SY, Kim SH. Clinical outcomes of transoral robotic surgery for head and neck tumors. Ann Otol Rhinol Laryngol 2013;122:73-84.
8. Aboul-Hosn Centenero S, Hernández-Alfaro F. 3D planning in orthognathic surgery: CAD/CAM surgical splints and prediction of the soft and hard tissues results - our experience in 16 cases. J Craniomaxillofac Surg 2012;40:162-8.

9. Li B, Zhang L, Sun H, Yuan J, Shen SG, Wang X. A novel method of computer aided orthognathic surgery using individual CAD/ CAM templates: a combination of osteotomy and repositioning guides. Br J Oral Maxillofac Surg 2013. [Epub ahead of print] 